\title{
Bevacizumab-based Chemotherapy for Poorly-differentiated Neuroendocrine Tumors
}

\author{
THOMAS COLLOT ${ }^{1,2,3,4}$, JEAN-DAVID FUMET ${ }^{1,2,3,4}$, QUENTIN KLOPFENSTEIN KN $^{1,3,4}$, \\ JULIE VINCENT ${ }^{2}$, LEILA BENGRINE $^{2}$ and FRANCOIS GHIRINGHELLI ${ }^{1,2,3,4}$ \\ ${ }^{1}$ University of Bourgogne Franche-Comté, Dijon, France; \\ ${ }^{2}$ Department of Medical Oncology and ${ }^{3}$ Platform of Transfer in Cancer Biology, \\ Georges-Francois Leclerc Centre, Dijon, France; \\ ${ }^{4}$ Centre de Recherche INSERM LNC-UMR1231, Dijon, France
}

\begin{abstract}
Aim: To assess and report the efficacy of and tolerance to bevacizumab-based chemotherapy in treatment outcome of metastatic poorly differentiated neuroendocrine tumors. Patients and Methods: From 2007 to 2018, 11 consecutive patients with metastatic poorly differentiated neuroendocrine treated in first- or second-line with bevacizumab-based chemotherapies were included in this monocentric retrospective cohort. Tumor response was evaluated by computed tomographic scans. Results: Administered treatment included 5-fluorouracil and irinotecan (FOLFIRI) bevacizumab, 5-fluorouracil and oxaliplatin (FOLFOX) bevacizumab and 5-fluorouracil, oxaliplatin and irinotecan (FOLFIRINOX) bevacizumab for four, two and five patients, respectively. Three were treated in first-line and eight in second-line after cisplatin-etoposide regimen. Using Response Evaluation Criteria in Solid Tumors, partial response was observed for seven patients, and stable disease for one patient, giving a response rate of $63.6 \% 195 \%$ confidence interval $=35.2-92.1 \%)$ and disease control rate of $72.7 \%$ (95\% confidence interval=46.6-99.0\%). All patients had died by the time of analysis, median progression-free survival was 14 months, and median overall survival was 15.3 months. Observed toxicity with such protocols was classical with 10 grade 3-4 toxic events, including three of hematological toxicity, three of infection, and three of digestive toxicity. Conclusion: Bevacizumab-based chemotherapy gave surprising efficacy and safety in first-or second-line treatment for metastatic poorly differentiated neuroendocrine tumor in
\end{abstract}

Correspondence to: Francois Ghiringhelli, MD, Ph.D., GeorgesFrancois Leclerc Cancer Center, 1 rue Professeur Marion, Dijon 21000, France. Tel: +33 380393353, Fax: +33 380393434, e-mail: fghiringhelli@cgfl.fr

Key Words: Metastatic poorly differentiated neuroendocrine tumor, bevacizumab, chemotherapy, retrospective study. this retrospective cohort. Prospective randomized trials of such therapy are warranted.

Neuroendocrine carcinomas (NECs) are a group of heterogeneous malignancies with a varied primary tumor location, histological type and prognosis and account for about $0.5 \%$ of newly-diagnosed cancers (1). The incidence is currently on the rise because of better detection by imaging, and is around 5/100,000 per year (1) with a higher incidence in females (2). The primary tumor location of NECs is heterogenous and involves many sites such as the central nervous system, lung, gastrointestinal tract, thyroid, skin, breast, and urogenital tract $(3,4)$. However, the gastroinstestinal tract, with $60 \%$ of cases, and lungs, with $25 \%$ of cases, are the most frequent tumor sites (5).

These tumors are classified using the fourth version of the WHO classification. Gastrointestinal NECs are subdivided into three groups according to their mitotic index or Ki67 expression (6).

Poorly differentiated NECs are a minority subgroup of digestive NECs which represents around $10 \%$ of these tumors $(3,7,8)$. These tumors can be found in the entire gastrointestinal tract, but are more frequently found in duodenopancreatic and colorectal localization. The prognosis for patients suffering from NECs remains poor, with median survival at around 11-17 months $(5,9,10)$. For metastatic disease, treatment is palliative using systemic chemotherapy. The rarity of this pathology explains the low level of proof for the American Society of Clinical Oncology recommendations. There are very few clinical trials and no phase III study. The most common first-line of chemotherapy consists of the association of etoposide and cisplatin. Such therapies lead to response rates of $40-70 \%(11,12)$. However, all patients will develop early secondary resistance and the median duration of the radiologicaI response to such therapy is short. At recurrence, no chemotherapeutic protocols have demonstrated clinical benefit in clinical trials. Some reports suggest that 
Table I. Patient characteristics $(n=11)$

\begin{tabular}{ll}
\hline Characteristic & Value \\
\hline WHO classification, $\mathrm{n}$ & \\
Grade 3 & 11 \\
$N C C N$ poorly differentiation status, $\mathrm{n}$ & 11 \\
Primary tumor location, $\mathrm{n}$ & \\
Duodenopancreatic & 2 \\
Colorectal & 4 \\
Other & 5 \\
Age, years & \\
Median (range) & 61.7 (41-79) \\
Gender, $\mathrm{n}$ & \\
Male & 6 \\
Female & 5 \\
ECOG performance status, $\mathrm{n}$ & \\
0 & 3 \\
1 & 8 \\
2 & 0 \\
NSE level, ng/mI & \\
Median (range) & 20.7 (9.7-246.7) \\
Metastasis at diagnosis, $\mathrm{n}$ & 10 \\
Liver metastasis, $\mathrm{n}$ & 11 \\
Previous chemotherapy, $\mathrm{n}$ & \\
Cisplatin-etoposide & 7 \\
Other & 1 \\
Protocol, $\mathrm{n}$ & \\
FOLFIRINOX + bevacizumab & 5 \\
FOLFIRI + bevacizumab & \\
FOLFOX + bevacizumab & \\
\hline
\end{tabular}

ECOG: Eastern Cooperative Oncology Group. NSE: Neuron specific enolase. NCCN: National Comprehensive Cancer Network.

temozolomide (13-15) and chemotherapeutic protocols used in colorectal cancer, such as 5-fluorouracil and oxaliplatin (FOLFOX) (16) or 5-fluorouracil and irinotecan (FOLFIRI) (17), induce response. NECs of both low and high grades are highly vascularized. Moreover, neoangiogenesis is more pronounced in poorly differentiated tumors (18). Antiangiogenic agents have demonstrated efficacy against well-differentiated tumors and drugs such as sunitinib or bevacizumab given in association with cytotoxic drugs has shown efficacy in clinical trials $(19,20)$. Based on these data and because of the low level of evidence for recommended treatment, patients with metastatic poorly differentiated NECs at our center were treated in first- or second-line like aggressive colorectal cancer with classical bi- or tri-chemotherapy for colorectal cancer with the addition of bevacizumab. In this article, we report the safety and efficacy of this strategy.

\section{Patients and Methods}

Eligibility criteria. All consecutive patients with metastatic histologically-proven poorly differentiated digestive NEC treated with bevacizumab-based chemotherapy at the Georges Francois
Table II. Observed toxicities according National Cancer Institute Common Terminology Criteria for grading of adverse events version 5.0 scale (https://ctep.cancer.gov/protocolDevelopment/electronic_ applications/ctc.htm) $(n=11)$.

\begin{tabular}{lccc}
\hline & \multicolumn{3}{c}{ NCI-CTC grade, $\mathrm{n}$} \\
\cline { 2 - 4 } & All grades & Grade 3 & Grade 4 \\
\hline Hematological & & & \\
Anemia & 7 & 1 & 0 \\
Neutropenia & 4 & 1 & 1 \\
Thrombocytopenia & 4 & 0 & 0 \\
Non-hematological & & & \\
Nausea/vomiting & 2 & 0 & 0 \\
Mucositis & 1 & 1 & 0 \\
Diarrhea & 5 & 2 & 0 \\
Neuropathy & 5 & 0 & 0 \\
Infection & 3 & 1 & 2 \\
Nose bleeding & 1 & 0 & 0 \\
High blood pressure & 1 & 0 & 0 \\
Proteinuria & 1 & 0 & 0 \\
Digestive hemorrhage & 1 & 0 & 1 \\
\hline
\end{tabular}

$\mathrm{N}$, Number of patients.

Leclerc Cancer Center from April 2007 to June 2018 were included in the study. Patients were retrospectively selected using the computer software of chemotherapy prescription $\left(\mathrm{CHIMIO}^{\circledR}\right.$, Computer Engineering, Paris France). All patients had either metastatic or locally advanced non-operable tumors based on the recommendation of a digestive surgeon. All patients received at least one cycle of bevacizumab-based therapy. The study was performed in accordance with standard procedures in France with approval by the ethical institutional Review Boards (N20180012). The database was declared to the National French Commission for bioinformatics data and patient liberty (CNIL). All patients give written consent at their first visit in the cancer center for research use of their clinical data. This consent allowed us to use their clinical and biological data in the cohort study.

Clinical, histological, biological, and radiological data [tumor response according to Response Evaluation Criteria in Solid Tumors (RECIST) v1.1 criteria (21)], and treatment outcomes were all collected in the medical records.

Treatment. The FOLFIRI plus bevacizumab regimen consisted of bevacizumab injection $(5 \mathrm{mg} / \mathrm{kg})$ followed by irinotecan $\left(180 \mathrm{mg} / \mathrm{m}^{2}\right.$ i.v. over $90 \mathrm{~min})$ concurrently with folinic acid $\left(400 \mathrm{mg} / \mathrm{m}^{2} i . v\right.$. over $120 \mathrm{~min}$ ), followed by fluorouracil bolus ( $400 \mathrm{mg} / \mathrm{m}^{2} i . v$. bolus) then continuous intravenous infusion of fluorouracil $\left(2,400 \mathrm{mg} / \mathrm{m}^{2}\right.$ over $46 \mathrm{~h})$. The FOLFOX plus bevacizumab regimen consisted of bevacizumab injection $(5 \mathrm{mg} / \mathrm{kg})$ followed by oxaliplatin $\left(85 \mathrm{mg} / \mathrm{m}^{2}\right.$ i.v. over $120 \mathrm{~min})$, followed by folinic acid $\left(400 \mathrm{mg} / \mathrm{m}^{2} i . v\right.$. over $120 \mathrm{~min})$ then fluorouracil bolus $\left(400 \mathrm{mg} / \mathrm{m}^{2}\right.$ i.v. bolus $)$ then continuous intravenous infusion of fluorouracil $\left(2,400 \mathrm{mg} / \mathrm{m}^{2}\right.$ over $46 \mathrm{~h}$ ). The FOLFIRINOX plus bevacizumab regimen consisted of bevacizumab injection $(5 \mathrm{mg} / \mathrm{kg})$ followed by oxaliplatin $\left(85 \mathrm{mg} / \mathrm{m}^{2}\right.$ i.v. over $120 \mathrm{~min})$, followed by irinotecan $\left(180 \mathrm{mg} / \mathrm{m}^{2} i . v\right.$. over 
$90 \mathrm{~min}$ ) concurrently with folinic acid $\left(400 \mathrm{mg} / \mathrm{m}^{2}\right.$ i.v. over $\left.120 \mathrm{~min}\right)$, followed by fluorouracil bolus ( $400 \mathrm{mg} / \mathrm{m}^{2} i . v$. bolus) then continuous intravenous infusion of fluorouracil $\left(2400 \mathrm{mg} / \mathrm{m}^{2}\right.$ over $\left.46 \mathrm{~h}\right)$. Adverse events were assessed using Common Terminology Criteria for Adverse Events (CTCAE) v4.0 (22).

Statistical analysis. Efficacy was evaluated according to the intention to-treat principle meaning that a patient was considered assessable for response if they had measurable disease, and had received at least one cycle of chemotherapy. All patients were followed-up until death or loss to follow-up. Progression-free survival (PFS) and overall survival (OS) were calculated using the Kaplan-Meier method. PFS was calculated from the start of bevacizumab to the date of disease progression upon computed tomographic scan, and OS was calculated from the start of bevacizumab to the date of death. The statistical software $\mathrm{R}$ (http://www.R-project.org/) was used for statistical analysis and graphical representations were made with Prism 7 (GraphPad, San Diego, CA, USA).

\section{Results}

Patient characteristics. Between April 2007 and June 2018, 11 patients were treated at our Center by bevacizumab therapy for metastatic poorly differentiated digestive neuroendocrine tumor. Three patients were treated in first line and seven in second line after cisplatin etoposide regimen. One patient was treated after Adriblastine Streptozotocine regimen. The most frequent location was the colorectum. Chemotherapeutic regimens were FOLFIRINOX, FOLFIRI and FOLFOX for five, four and two patients, respectively. Table I shows the patients' clinical characteristics.

Toxicity and feasibility. A total of 180 cycles of bevacizumabbased chemotherapy were administered (median $=15$; range $=3$ 38). The median number of cycles of FOLFIRINOX, FOLFIRI and FOLFOX was 19, 15 and 14.5, respectively. The median number of cycles in first line was 20 and in second line was 15 .

Table II gives information on hematological and nonhematological toxicities. Grade 3 and 4 toxic events were observed in 10 patients. Two cases of febrile neutropenia were observed. A chemotherapy dose reduction or delay was required for six patients because of hematological toxicity for two, diarrhea for one, neuropathy for two and infection for one.

Concerning bevacizumab-induced toxicity, no serious adverse events were observed. Indeed, we did not observe bowel perforation or brain bleeding. One patient presented acute high digestive hemorrhage linked to esophageal varices on cirrhosis. High blood pressure and epistaxis occurred in two patients.

At the time of analysis, with a median follow-up of 21.2 months (range=5.1-53.3 months), all patients had died because of disease progression.

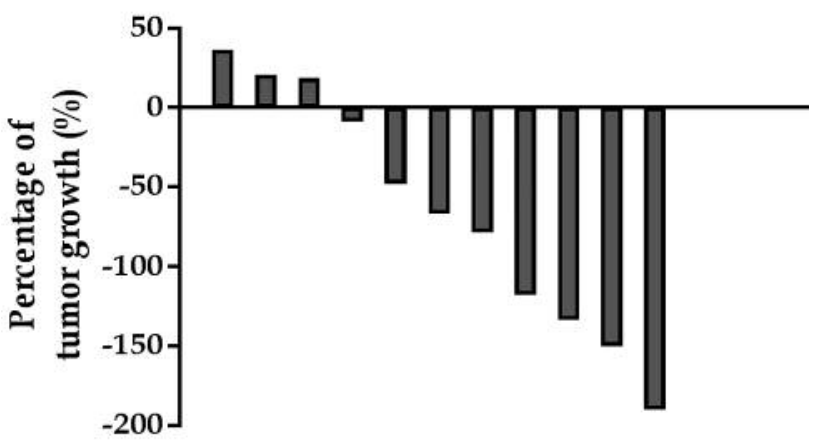

Figure 1. Change in tumor size at the best response for the whole cohort of 11 patients with poorly differentiated neuroendocrine carcinomas treated with bevacizumab-based regimen.

Objective tumor responses and survival. According to RECIST, we recorded no complete response, seven partial responses, one case with stable disease and three with progressive disease as best response. The response rate was, therefore, $63.6 \%$ (95\% confidence interval $=35.2-92.1 \%)$ and the disease control rate (partial responses + stable diseases) was $72.7 \%$ (95\% confidence interval $=46.6-99.0 \%)$. Figure 1 represents the percentages of tumor growth at the best response of each patient. For the whole cohort, PFS was a median of 14 months (Figure 2A), and median OS was 15.3 months (Figure 2B). Clinical responses for all patients are summarized in Table III.

\section{Discussion}

The treatment of metastatic poorly differentiated digestive neuroendocrine tumor currently remains a challenging question. These tumors require urgent systemic chemotherapy. The most frequently used protocol combines platinum salts (cisplatin or carboplatin) with etoposide. NECs are highly sensitive to such cytotoxic drugs, with response rates ranging from $40 \%$ to $75 \%$. However, the duration of treatment response is short and all patients rapidly experience relapse and develop some resistance leading to a median OS of around 15-18 months. The levels of evidence for such protocol are, however, poor and only based on old retrospective studies $(3,11)$.

No protocol is currently validated for use after tumor progression. Based on the efficacy of temozolomide for welldifferentiated tumors, protocols using temozolomide alone or its combination with capecitabine were proposed. Response rates were around $30 \%$, with 6 months PFS (13). By analogy to small cell lung cancer, topotecan was proposed as a possible agent in second-line therapy, but no study has tested this hypothesis. Some retrospective reports also proposed that FOLFIRI or FOLFOX could be used in this setting (16, 

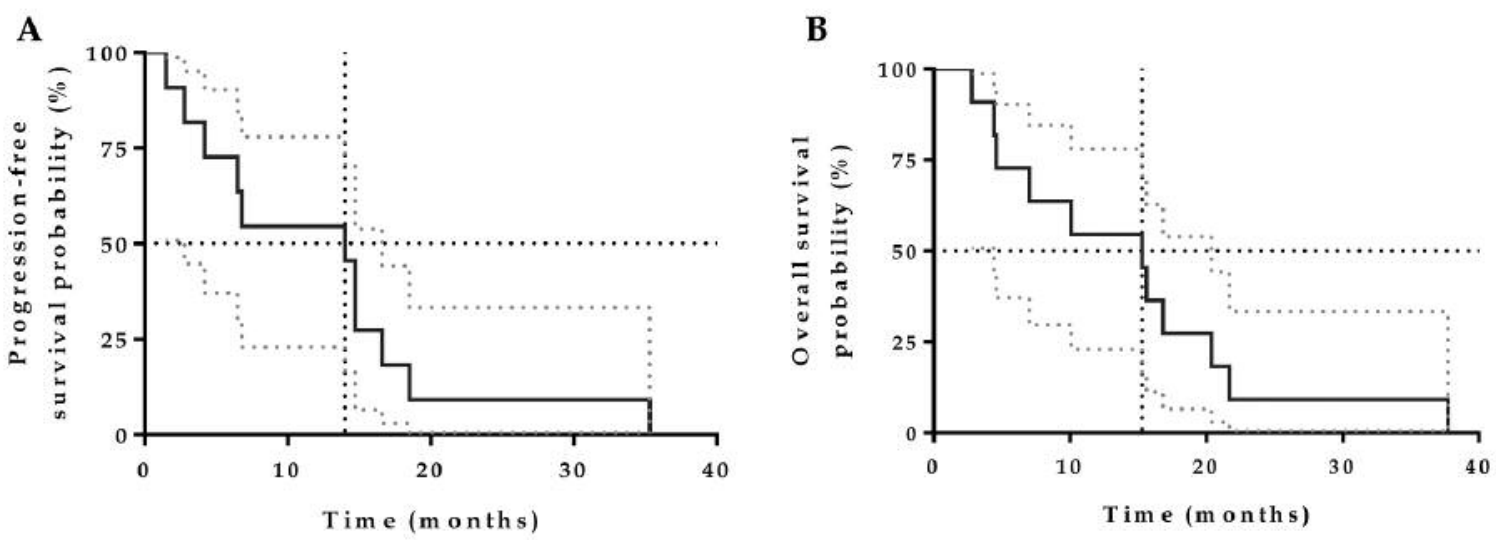

Figure 2. Progression-free (A) and overall (B) survival of the 11 patients with poorly differentiated neuroendocrine carcinomas of our cohort treated with bevacizumab-based regimen.

Table III. Individual clinical response.

\begin{tabular}{|c|c|c|c|c|c|c|c|}
\hline \multirow[b]{2}{*}{ Patient } & \multirow[b]{2}{*}{$\begin{array}{l}\text { Previous } \\
\text { therapy }\end{array}$} & \multirow[b]{2}{*}{$\begin{array}{l}\text { Treatment received } \\
(\mathrm{Bev}+)\end{array}$} & \multirow[b]{2}{*}{$\begin{array}{c}\text { Best } \\
\text { response }\end{array}$} & \multirow[b]{2}{*}{$\begin{array}{l}\text { Cycles } \\
\text { of Bev }\end{array}$} & \multirow[b]{2}{*}{$\begin{array}{l}\text { PFS line } \\
\text { (months) }\end{array}$} & \multicolumn{2}{|c|}{ OS } \\
\hline & & & & & & $\begin{array}{c}\text { From first } \\
\text { (months) }\end{array}$ & $\begin{array}{r}\text { From Bev } \\
\text { (months) }\end{array}$ \\
\hline 1 & Adriamycin+streptozocin & FOLFOX & PR & 26 & 16.6 & 36.6 & 21.7 \\
\hline 2 & Carboplatin+etoposide & FOLFOX & $\mathrm{PD}$ & 3 & 1.5 & 5.1 & 2.8 \\
\hline 3 & Cisplatin+etoposide & FOLFIRI & PR & 15 & 6.8 & 15.6 & 10.1 \\
\hline 4 & Cisplatin+etoposide & FOLFIRI & $\mathrm{PD}$ & 7 & 4.2 & 14.5 & 4.6 \\
\hline 5 & Cisplatin+etoposide & FOLFIRI & PR & 15 & 14.7 & 46.3 & 15.3 \\
\hline 6 & Cisplatin+etoposide & FOLFIRI & PR & 38 & 35.3 & 53.3 & 37.7 \\
\hline 7 & Cisplatin+etoposide & FOLFIRINOX & PD & 4 & 2.8 & 22.8 & 4.4 \\
\hline 8 & Cisplatin+etoposide & FOLFIRINOX & PR & 19 & 14.0 & 31.6 & 16.8 \\
\hline 9 & None & FOLFIRINOX & PR & 10 & 6.5 & 7.0 & 7.0 \\
\hline 10 & None & FOLFIRINOX & PR & 23 & 14.7 & 15.6 & 15.6 \\
\hline 11 & None & FOLFIRINOX & SD & 20 & 18.5 & 20.4 & 20.4 \\
\hline
\end{tabular}

PR: Partial response; SD: stable disease; PD: progressive disease; PFS: progression-free survival; OS: overall survival; Bev: bevacizumab.

17). Such regimens give response in around $30 \%$ of patients, with PFS of about 4 months and OS of around 10 months.

More recently, the efficacy of first- and second-line therapy was retrospectively evaluated in a large French national cohort which included 253 patients. First- and second-line palliative chemotherapies for metastatic NECs were given in $176(69 \%)$ and $100(40 \%)$ patients. OS from the beginning of first-line and second-line palliative chemotherapy were $12.1(10.1-13.7)$ and 7.2 (5.3-9.0) months, respectively. Among patients treated in first line, $90 \%$ received cisplatin plus etoposide or carboplatin plus etoposide regimen. Such therapy gave an overall response rate of $50 \%$, PFS of 6 (5.2-7.6) months and OS of 11.6 (9.213.5) months from start of the chemotherapy. In second line, patients mainly received FOLFIRI $(n=72)$ or FOLFOX $(n=33)$. About $20 \%$ of patients experienced response and about $40 \%$ of patients had controlled disease as best response. However, PFS was short at between 2 and 3 months. OS was 5.9 (4.9-7.6) months for patients that received FOLFIRI and 3.9 (2.2-8.9) months for patients treated with FOLFOX (9).

Most NECs are hypervascular, this is one of their classical characteristics for imaging diagnostics. Some studies have shown that intratumoral microvascular density is more important in NECs than in carcinomas (23-25). NEC cells frequently synthesize vascular endothelial growth factor $\mathrm{A}$ (VEGFA) or other VEGF molecules such as VEGFC and VEGFD (26) and consequently this can promote tumor 
neoangiogenesis. In addition, more than $40 \%$ of NECs contain elements of non-neuroendocrine histology. In the WHO classification of digestive tumors, mixed neuroendocrine-nonneuroendocrine neoplasm is considered as a specific type and is defined as mixed adenoneuroendocrine carcinoma (27). Such data raise the hypothesis that in some cases, neuroendocrine tumors may arise from dedifferentiation of an adenocarcinoma component. These data suggest that such tumors may gain benefit from similar therapy than those given in digestive adenocarcinomas.

At our center, it was decided to propose bevacizumabbased chemotherapy in first- or second-line for metastatic poorly differentiated digestive neuroendocrine tumor. Because of the aggressiveness of this tumor type, the classical regimen proposed is FOLFIRINOX bevacizumab, which was demonstrated to give a higher response rate than the combination of bi-chemotherapy plus bevacizumab in metastatic colorectal cancer (28). Patients were only treated with bevacizumab in combination with bi-chemotherapy when the clinician had contraindications for FOLFIRINOX usage. This strategy was validated after multidisciplinary staff, and all patients gave their written consent. We also examined the efficacy and toxicity of the protocol for each patient. These regimens gave high response rates in both first and second lines. PFS was 14 months and compared favorably to cisplatin-etoposide as first line and FOLFOX/FOLFIRI as second line. However, due to the monocentric design and the small number of patients of this study, such data must be confirmed by other studies.

Limitations of this study were its retrospective and noncomparative design, and the low number of patients. However, few prospective studies have been performed to assess efficacy of new chemotherapeutic regimens for poorly differentiated NECs. In addition, in the literature we did not find other prospective or retrospective studies that address the antitumoral effect of combination of chemotherapy plus antiangiogenic agent in poorly differentiated NECs. In France, a prospective randomized phase II study which will compare second-line FOLFIRI versus FOLFIRI plus bevacizumab will start in 2018 to address this question.

In conclusion, bevacizumab-based chemotherapy using FOLFIRINOX or FOLFOX or FOLFIRI regimen showed antitumor efficacy and safety as first- or second-line treatment for metastatic poorly differentiated NECs. Larger-scale prospective trials are ongoing to confirm this conclusion.

\section{Conflicts of Interest}

F. Ghiringhelli received honoraria for oral communication from Lilly, Sanofi and Amgen and is an advisory board member for Merck Serano, Amgen, Sanofi. No other Author has any potential conflict of interest to disclose.

\section{References}

1 Taal BG and Visser O: Epidemiology of neuroendocrine tumours. Neuroendocrinology 80: 3-7, 2004.

2 Rindi G, Bordi C, Rappel S, La Rosa S, Stolte M and Solcia E: Gastric carcinoids and neuroendocrine carcinomas: pathogenesis, pathology, and behavior. World J Surg 20: 168-172, 1996.

3 Niederle MB, Hackl M, Kaserer $\mathrm{K}$ and Niederle B: Gastroenteropancreatic neuroendocrine tumours: the current incidence and staging based on the WHO and European Neuroendocrine Tumour Society classification: an analysis based on prospectively collected parameters: Endocr Relat Cancer 17: 909-918, 2010.

4 Modlin IM, Oberg K, Chung DC, Jensen RT, de Herder WW, Thakker RV, Caplin M, Delle Fave G, Kaltsas GA, Krenning EP, Moss SF, Nilsson O, Rindi G, Salazar R, Ruszniewski P and Sundin A: Gastroenteropancreatic neuroendocrine tumours. Lancet Oncol 9: 61-72, 2008.

5 Heetfeld M, Chougnet CN, Olsen IH, Rinke A, Borbath I, Crespo G, Barriuso J, Pavel M, O'Toole D, Walter T and other Knowledge Network members: Characteristics and treatment of patients with G3 gastroenteropancreatic neuroendocrine neoplasms. Endocr Relat Cancer 22: 657-664, 2015.

6 Kulke MH, Shah MH, Benson AB, Bergsland E, Berlin JD, Blaszkowsky LS, Emerson L, Engstrom PF, Fanta P, Giordano T, Goldner WS, Halfdanarson TR, Heslin MJ, Kandeel F, Kunz PL, Kuvshinoff BW, Lieu C, Moley JF, Munene G, Pillarisetty VG, Saltz L, Sosa JA, Strosberg JR, Vauthey J-N, Wolfgang C, Yao JC, Burns J and Freedman-Cass D: National comprehensive cancer network. Neuroendocrine tumors, version 1.2015. J Natl Compr Cancer Netw JNCCN 13: 78-108, 2015.

7 Lepage C, Bouvier AM, Phelip JM, Hatem C, Vernet C and Faivre J: Incidence and management of malignant digestive endocrine tumours in a well defined French population. Gut 53: 549-553, 2004

8 Yao JC, Hassan M, Phan A, Dagohoy C, Leary C, Mares JE, Abdalla EK, Fleming JB, Vauthey J-N, Rashid A and Evans DB: One hundred years after 'carcinoid': epidemiology of and prognostic factors for neuroendocrine tumors in 35,825 cases in the United States. J Clin Oncol Off J Am Soc Clin Oncol 26: 3063-3072, 2008.

9 Walter T, Tougeron D, Baudin E, Le Malicot K, Lecomte T, Malka D, Hentic O, Manfredi S, Bonnet I, Guimbaud R, Coriat $\mathrm{R}$, Lepère C, Desauw C, Thirot-Bidault A, Dahan L, Roquin G, Aparicio T, Legoux J-L, Lombard-Bohas C, Scoazec J-Y, Lepage $\mathrm{C}$ and Cadiot G: Poorly differentiated gastro-entero-pancreatic neuroendocrine carcinomas: Are they really heterogeneous? Insights from the FFCD-GTE national cohort. Eur J Cancer 79: 158-165, 2017.

10 Sorbye H, Welin S, Langer SW, Vestermark LW, Holt N, Osterlund P, Dueland S, Hofsli E, Guren MG, Ohrling K, Birkemeyer E, Thiis-Evensen E, Biagini M, Gronbaek H, Soveri LM, Olsen IH, Federspiel B, Assmus J, Janson ET and Knigge U: Predictive and prognostic factors for treatment and survival in 305 patients with advanced gastrointestinal neuroendocrine carcinoma (WHO G3): The NORDIC NEC study. Ann Oncol 24: 152-160, 2013.

11 Moertel CG, Kvols LK, O'Connell MJ and Rubin J: Treatment of neuroendocrine carcinomas with combined etoposide and cisplatin. Evidence of major therapeutic activity in the anaplastic variants of these neoplasms. Cancer 68: 227-232, 1991. 
12 Mitry E, Baudin E, Ducreux M, Sabourin J-C, Rufié P, Aparicio T, Lasser P, Elias D, Duvillard P, Schlumberger M and Rougier P: Treatment of poorly differentiated neuroendocrine tumours with etoposide and cisplatin. Br J Cancer 81: 1351-1355, 1999.

13 Welin S, Sorbye H, Sebjornsen S, Knappskog S, Busch C and Oberg K: Clinical effect of temozolomide-based chemotherapy in poorly differentiated endocrine carcinoma after progression on first-line chemotherapy. Cancer 117: 4617-4622, 2011.

14 Olsen IH, Sørensen JB, Federspiel B, Kjaer A, Hansen CP, Knigge $U$ and Langer SW: Temozolomide as Second or Third Line Treatment of Patients with Neuroendocrine Carcinomas. Sci World J 2: 170496, 2012.

15 Lindholm DP, Eriksson B and Granberg D: Response to temozolomide and bevacizumab in a patient with poorly differentiated neuroendocrine carcinoma. Med Oncol 29: 301303, 2012.

16 Hadoux J, Malka D, Planchard D, Scoazec JY, Caramella C, Guigay J, Boige V, Leboulleux S, Burtin P, Berdelou A, Loriot Y, Duvillard P, Chougnet CN, Déandréis D, Schlumberger M, Borget I, Ducreux M and Baudin E: Post-first-line FOLFOX chemotherapy for grade 3 neuroendocrine carcinoma. Endocr Relat Cancer 22: 289-298, 2015.

17 Hentic O, Hammel P, Couvelard A, Rebours V, Zappa M, Palazzo M, Maire F, Goujon G, Gillet A, Lévy P and Ruszniewski P: FOLFIRI regimen: an effective second-line chemotherapy after failure of etoposide-platinum combination in patients with neuroendocrine carcinomas grade 3. Endocr Relat Cancer 19: 751-757, 2012.

18 Scoazec J-Y: Angiogenesis in neuroendocrine tumors: therapeutic applications. Neuroendocrinology 97: 45-56, 2013.

19 Ducreux M, Dahan L, Smith D, O'Toole D, Lepère C, Dromain C, Vilgrain V, Baudin E, Lombard-Bohas C, Scoazec J-Y, Seitz J-F, Bitoun L, Koné S and Mitry E:. Bevacizumab combined with 5-FU/streptozocin in patients with progressive metastatic well-differentiated pancreatic endocrine tumours (BETTER trial)--a phase II non-randomised trial. Eur J Cancer 50: 30983106, 2014.

20 Mitry E, Walter T, Baudin E, Kurtz J-E, Ruszniewski P, Dominguez-Tinajero S, Bengrine-Lefevre L, Cadiot G, Dromain C, Farace F, Rougier P and Ducreux M: Bevacizumab plus capecitabine in patients with progressive advanced welldifferentiated neuroendocrine tumors of the gastro-intestinal (GINETs) tract (BETTER trial)-a phase II non-randomised trial. Eur J Cancer 50: 3107-3115, 2014.

21 Eisenhauer EA, Therasse P, Bogaerts J, Schwartz LH, Sargent D, Ford R, Dancey J, Arbuck S, Gwyther S, Mooney M, Rubinstein L, Shankar L, Dodd L, Kaplan R, Lacombe D and Verweij J: New response evaluation criteria in solid tumours: Revised RECIST guideline (version 1.1). Eur J Cancer 45: 228247, 2009.
22 National Cancer Institute, National Institutes of Health, US Department of Health and Human Services: Common Terminology Criteria for Adverse Events (CTCAE) (version 4.0). Bethesda, MD, National Cancer Institute, NIH publication 09-7473. 2009; Revised version 4.03 June 14, 2010. https://evs.nci.nih.gov/ftp1/CTCAE/CTCAE_4.03

23 Chen J, Deng Q, Pan Y, He B, Ying H, Sun H, Liu X and Wang S: Prognostic value of neutrophil-to-lymphocyte ratio in breast cancer. FEBS Open Bio 5: 502-507, 2015.

24 Couvelard A, O'Toole D, Turley H, Leek R, Sauvanet A, Degott C, Ruszniewski P, Belghiti J, Harris AL, Gatter K and Pezzella F: Microvascular density and hypoxia-inducible factor pathway in pancreatic endocrine tumours: negative correlation of microvascular density and VEGF expression with tumour progression. Br J Cancer 92: 94-101, 2005.

25 Marion-Audibert AM, Barel CC, Gouysse G, Dumortier J, Pilleul F, Pourreyron C, Hervieu V, Poncet G, Lombard-Bohas C, Chayvialle JA, Partensky C and Scoazec JY: Low microvessel density is an unfavorable histoprognostic factor in pancreatic endocrine tumors. Gastroenterology 125: 1094-1104, 2003.

26 Christofori G, Naik P and Hanahan D: Vascular endothelial growth factor and its receptors, flt-1 and flk-1, are expressed in normal pancreatic islets and throughout islet cell tumorigenesis. Mol Endocrinol 9: 1760-1770, 1995.

27 La Rosa S, Sessa F and Uccella S: Mixed NeuroendocrineNonneuroendocrine Neoplasms (MiNENs): Unifying the Concept of a Heterogeneous Group of Neoplasms. Endocr Pathol 27: 284-311, 2016.

28 Loupakis F, Cremolini C, Masi G, Lonardi S, Zagonel V, Salvatore L, Cortesi E, Tomasello G, Ronzoni M, Spadi R, Zaniboni A, Tonini G, Buonadonna A, Amoroso D, Chiara S, Carlomagno C, Boni C, Allegrini G, Boni L and Falcone A: Initial therapy with FOLFOXIRI and bevacizumab for metastatic colorectal cancer. N Engl J Med 371: 1609-1618, 2014.
Received August 30, 2018

Revised September 14, 2018

Accepted September 17, 2018 